\title{
The 2019 Lasker Award: T cells and B cells, whose life and death are essential for function of the immune system
}

\author{
Daniel H. D. Gray $\mathbb{1}^{1,2} \cdot$ David L. Vaux $\mathbb{B}^{1,2} \cdot$ Andreas Strasser ${ }^{1,2}$
}

Received: 22 September 2019 / Accepted: 23 September 2019 / Published online: 17 October 2019

(c) The Author(s), under exclusive licence to ADMC Associazione Differenziamento e Morte Cellulare 2019

Before 1960, the Bursa of Fabricius was known to be important for development of lymphocytes in chickens, but the analogous organ in mammals was unknown. At that time the thymus was generally thought to be an "evolutionary accident" of little consequence; merely a graveyard for lymphocytes. Now, the concept that $\mathrm{T}$ and $\mathrm{B}$ cells are distinct, and must collaborate for adaptive immune responses, is entrenched in textbooks, and underpins therapies to combat infection, organ rejection, autoimmune diseases, immune-deficiencies and cancer. The 2019 Albert Lasker Basic Medical Research Award recognises the ground-breaking discoveries made by Jacques Miller and Max Cooper who defined the anatomical origins of the two arms of adaptive immunity, the specialisation of $\mathrm{T}$ and $\mathrm{B}$ cells for host defence, and the nature of the collaboration between these cell types to mount effective immune responses. In the decades since these discoveries, $\mathrm{T}$ and $\mathrm{B}$ cell development has been a fertile ground for research in cell death and differentiation.

In 1960, it was known that the thymus was full of lymphocytes, but it was thought to be irrelevant to immunity because thymectomy of adult mice had no measurable impact. Jacques Miller, working in converted stables at the Institute of Cancer Research in the UK, mastered the surgical removal of the thymus from newborn mice in order to probe the nature of a virus-induced leukaemia. To his surprise, he found that neonatally thymectomized mice succumbed to fatal infections, were deficient in "small" lymphocytes, had impaired antibody production, and could

Daniel H. D. Gray

dgray@wehi.edu.au

$\triangle$ Andreas Strasser

strasser@wehi.edu.au

1 The Walter and Eliza Hall Institute of Medical Research, 1G Royal Parade, Parkville, VIC 3052, Australia

2 Department of Medical Biology, The University of Melbourne, Parkville, VIC 3010, Australia not reject foreign skin grafts $[1,2]$. He also discovered that thymic grafts from genetically distinct mice could not only restore immune function, but also engender tolerance to skin grafts from the donor origin $[1,3]$. This stunning series of single author papers established the immunological function of the thymus and its role in distinguishing self from non-self.

The concept that the thymus is an essential immune organ took some time to become accepted. Some groups had difficulty reproducing Miller's findings, in part because neonatal thymectomy is a challenging surgical technique, but also because some mature $T$ cells could emerge from the thymus in the few days prior to its removal. This latter problem also confounded replication of earlier experiments, showing that removal of the bursa of Fabricius from chickens impaired the production of antibodies [4]. Max Cooper, working in Robert Good's laboratory, solved this problem by irradiating chicks shortly after removal of the thymus and/or bursa to destroy residual mature lymphocytes. With this innovation, Cooper provided clear evidence that the bursa was essential for antibody production in response to immunisation [5, 6]. In addition, he replicated Miller's findings by showing that thymectomized and irradiated chickens were deficient in graft-versus-host responses, allograft rejection and hypersensitivity reactions $[5,6]$. Importantly, Cooper (a paediatrician) and his colleagues noted the striking parallels between the distinct immunodeficiencies observed in their bursectomized or thymectomized chickens and those manifest in primary immunodeficiencies in children-a crucial clinical insight.

Subsequent work from Jacques Miller and his PhD student Graham Mitchell demonstrated that this distinction in lymphocyte immune function also existed in mice, where the bone marrow was the origin of antibody-producing cells [7-10]. With this insight, they used neonatally thymectomized, irradiated F1 mice as vehicles to reconstitute these distinct arms of adaptive immunity, combining genetically distinct bone marrow cells with thoracic duct lymphocytes (of which $\sim 90 \%$ are $\mathrm{T}$ cells). They could then immunise the 
chimaeras with sheep red blood cells and determine the cellular source of antibodies in plaque assays by using antisera to ablate the genetically disparate bone marrowderived lymphocytes (B cells) or thoracic duct lymphocytes ( $\mathrm{T}$ cells). The results of these elegant experiments established that B cells were the antibody-producing cells, butcrucially - that they required "help" from thymus-derived T lymphocytes for optimal immune responses [7].

Once again, these discoveries took some time to become generally accepted. There was criticism that the data generated from such "biological monstrosities" as haematopoietic chimaeras was of questionable significance. A less diplomatic Australian scientist expressed the view that the only significance of "B" and "T" was that they represented the first and last letters of the word "bullshit". More reasonable arguments cited that $\mathrm{B}$ and $\mathrm{T}$ cells were so morphologically similar that they could not have such distinct functions. Furthermore, it was known that thymocytes were ultimately derived from bone marrow precursors, leading to further doubt. Finally, the concept that rare, antigen-specific $\mathrm{T}$ cells should somehow scan the entire body to find B cells that have been activated by the same antigen strained plausibility. In over 50 years of subsequent research, it has become apparent that the thymus provides a unique microenvironment that directs the differentiation of bone marrow-derived progenitors into $\mathrm{T}$ cells and that the architecture of secondary lymphoid organs orchestrates efficient interactions between highly mobile antigen-specific $\mathrm{B}$ and $\mathrm{T}$ cells.

Fig. 1 Schematic diagram of T- and B-cell differentiation, showing the major stages and apoptotic checkpoints mediated by either the intrinsic or extrinsic pathways
Lymphocyte biology has since become one of the most well-studied mammalian differentiation paradigms and one of the key models for studying cell death. Technological advances in flow cytometry, genetically modified mouse models, in vitro differentiation systems and deep genetic analyses, to name a few, have allowed researchers to detail the precise precursor-product relationships and critical checkpoints in T- and B-cell differentiation in the thymus and bone marrow, respectively. These studies have revealed largely parallel pathways, with some key distinctions mostly based on the presumptive logic of B-cell receptor versus T-cell receptor (TCR) (Fig. 1). For instance, MHC restriction of T-cell responses necessitate the positive selection of thymocytes that express a TCR capable of interacting with self-MHC/peptide complexes, while those incapable of binding undergo apoptosis [11]. Conversely, mature B cells are uniquely capable of further somatic mutation of rearranged immunoglobulin genes through the activity of AID during the germinal centre reaction to improve antibody affinity for antigen.

Both T- and B-cell differentiation are dynamic processes punctuated by high rates of apoptosis that purges useless cells or potentially dangerous cells that bear autoreactive immunoreceptors (e.g. reviewed in [12]) (Fig. 1). Accordingly, inhibition of apoptosis has major impacts on lymphocyte differentiation and homoeostasis, and leads to autoimmune disease by allowing the aberrant survival of autoreactive $\mathrm{T}$ and $\mathrm{B}$ cells as well as persistence of

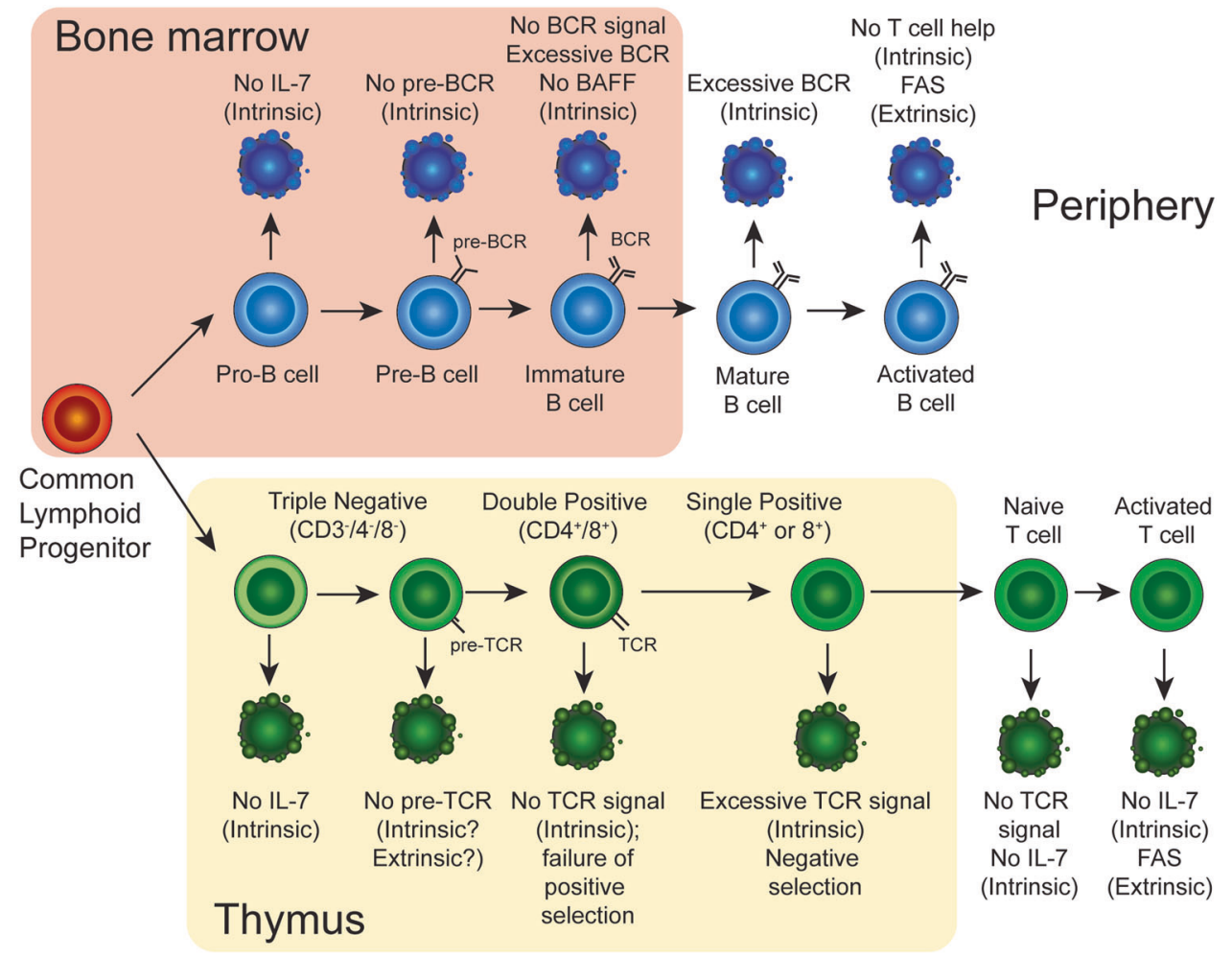


antigen-activated effector lymphocytes (e.g. plasma cells) $[13,14]$. Distinct apoptotic mechanisms are engaged at the major checkpoints of lymphocyte differentiation, providing insights into the divergence of the BCL-2-regulated (or mitochondrial or intrinsic) and death receptor (or extrinsic) pathways of apoptosis [15] (Fig. 1).

The discoveries of Jacques Miller and Max Cooper have revealed many of the secrets of the immune system, laying the foundations for subsequent breakthroughs in understanding the generation of antibody diversity (Tonegawa), hybridoma technology (Koehler and Milstein), MHC restriction (Benacerraf, Doherty and Zinkernagel), the isolation and cloning of the TCR (Tak Mak, Mark Davis, John Kappler and Pippa Marrack) and immune regulation (Steinman). In doing so, Miller and Cooper provided concepts and model systems for investigating the physiological and pathological roles of cell death. Indeed, Kerr et al. drew on the death of lymphocytes in germinal centres of lymphoid follicles, and in response to treatment with glucocorticoids, as exemplars of "apoptosis", the term they introduced in their influential 1972 review [16].

Acknowledgements DHDG is supported by an Australia NHMRC Senior Research Fellowship and project grants from the NHMRC (\#1158024, 1121325, 1145888) and Cancer Council Victoria (1146518). DLV is supported by NHMRC grants 1135864 and 1113133. AS is supported by fellowships and grants from the NHMRC (1116937, 1113133, and 1143105), the Cancer Council Victoria (1102104) a Leukemia \& Lymphoma Society Special Center of Research (SCOR 7015-18) and a bequest from the Estate of Anthony Redstone. This work was made possible through Victorian State Government Operational Infrastructure Support and Australian Government NHMRC IRIISS.

\section{Compliance with ethical standards}

Conflict of interest The authors declare that they have no conflict of interest.

Publisher's note Springer Nature remains neutral with regard to jurisdictional claims in published maps and institutional affiliations.

\section{References}

1. Miller JF. Immunological function of the thymus. Lancet. 1961;2:748-9.

2. Miller JF. Analysis of the thymus influence in leukaemogenesis. Nature. 1961;191:248-9.

3. Miller JF. Effect of neonatal thymectomy on the immunological responsiveness of the mouse. Proc R Soc Lond B Sci. 1962;156: 415-28.

4. Glick B, Chang TS, Jaap RG. The bursa of fabricius and antibody production. Poult Sci. 1956;35:224-5.

5. Cooper MD, Peterson RD, Good RA. Delineation of the thymic and bursal lymphoid systems in the chicken. Nature. 1965;205:143-6.

6. Cooper MD, Raymond DA, Peterson RD, South MA, Good RA. The functions of the thymus system and the bursa system in the chicken. J Exp Med. 1966;123:75-102.

7. Miller JF, Mitchell GF. The thymus and the precursors of antigen reactive cells. Nature. 1967;216:659-63.

8. Mitchell GF, Miller JF. Cell to cell interaction in the immune response. II. The source of hemolysin-forming cells in irradiated mice given bone marrow and thymus or thoracic duct lymphocytes. J Exp Med. 1968;128:821-37.

9. Miller JF, Mitchell GF. Cell to cell interaction in the immune response. I. Hemolysin-forming cells in neonatally thymectomized mice reconstituted with thymus or thoracic duct lymphocytes. J Exp Med. 1968;128:801-20.

10. Mitchell GF, Miller JF. Immunological activity of thymus and thoracic-duct lymphocytes. Proc Natl Acad Sci USA. 1968;59:296-303.

11. Strasser A, Harris AW, Corcoran LM, Cory S. Bcl-2 expression promotes B- but not T-lymphoid development in scid mice. Nature. 1994;368:457-60.

12. Daley SR, Teh C, Hu DY, Strasser A, Gray DHD. Cell death and thymic tolerance. Immunol Rev. 2017;277:9-20.

13. Strasser A, Whittingham S, Vaux DL, Bath ML, Adams JM, Cory $\mathrm{S}$, et al. Enforced BCL2 expression in B-lymphoid cells prolongs antibody responses and elicits autoimmune disease. Proc Natl Acad Sci USA. 1991;88:8661-5.

14. Rieux-Laucat F, Le Deist F, Hivroz C, Roberts IA, Debatin KM, Fischer A, et al. Mutations in Fas associated with human lymphoproliferative syndrome and autoimmunity. Science. 1995;268:1347-9.

15. Marsden VS, Strasser A. Control of apoptosis in the immune system: Bcl-2, BH3-only proteins and more. Annu Rev Immunol. 2003;21:71-105.

16. Kerr JF, Wyllie AH, Currie AR. Apoptosis: a basic biological phenomenon with wide-ranging implications in tissue kinetics. $\mathrm{Br}$ J Cancer. 1972;26:239-57. 